\title{
[Fell] as a tracer of supernova rate in nearby starburst galaxies
}

\author{
M. J. F. Rosenberg, P. P. van der Werf, and F. P. Israel
}

Sterrewacht Leiden, Universiteit Leiden, PO Box 9513, 2300 RA Leiden, The Netherlands

e-mail: rosenberg@strw. leidenuniv.nl

Received 4 January 2012 / Accepted 9 February 2012

\begin{abstract}
Context. Supernovae play an integral role in the feedback of processed material into the ISM of galaxies making them responsible for much of the chemical enrichment of the universe. The rate of supernovae can also reveal star formation histories. These rates are usually measured through the nonthermal radio continuum luminosity; however, a correlation between near-infrared [FeII] emission and supernova remnants has also been noted.

Aims. We aim to find a quantitative relationship between the $[\mathrm{FeII}]$ at $1.26 \mu \mathrm{m}\left([\mathrm{FeII}]_{1.26}\right)$ luminosity and the supernova rate in a sample of 11 nearby starburst galaxy centers.

Methods. We performed a pixel-pixel analysis of this correlation on SINFONI data cubes. Using Br $\gamma$ equivalent width and luminosity as the only observational inputs into the Starburst 99 model, we derived the supernova rate at each pixel and have thus created maps of supernova rates. We then compared these morphologically and quantitatively to the $[\mathrm{FeII}]_{1.26}$ luminosity.

Results. We have found that a strong linear and morphological correlation exists between supernova rate and $[\mathrm{Fe} I \mathrm{II}]_{1.26}$ on a pixel-topixel basis. This relation is valid for normal star-forming galaxies but breaks down for extreme ultraluminous galaxies.

Conclusions. The supernova rates derived from the Starburst 99 model are in good agreement with the radio derived supernova rates, which underlines the strength of using [FeII] emission as a tracer of supernova rate. With the strong correlation found in this sample of galaxies, we conclude that $[\mathrm{FeII}]_{1.26}$ emission can generally be used to derive accurate supernova rates on either a pixel-pixel or integrated galactic basis.
\end{abstract}

Key words. supernovae: general - ISM: supernova remnants - galaxies: nuclei - galaxies: ISM - galaxies: star formation infrared: galaxies

\section{Introduction}

Supernova rates (SNrates) are typically estimated by the integrated nonthermal radio continuum emission from their remnants (Condon 1992, and references therein). The connection between radio continuum emission and SNrate comes from the tight infrared-radio relation that is usually interpreted to be a natural consequence of (massive) star formation and stellar evolution (Condon 1992, and references therein). Observations of nearby starburst galaxies show many compact radio sources, generally attributed to supernova remnants (SNR). Condon \& Yin (1990) use observations of SNR in our own galaxy to establish a relation between the nonthermal radio continuum and SNrate. Huang et al. (1994) find a similar relation using observations of compact radio sources in M 82 .

In addition, near-infrared (NIR) observations of SNR often show strong [FeII] emission line flux at 1.257 and $1.644 \mu \mathrm{m}$ coincident with the radio peak (Graham et al. 1987, 1990; Oliva et al. 1989, 1990). In interstellar space, iron atoms are typically locked in dust grains. However, shock fronts associated with SNRs cause efficient grain destruction through thermal sputtering. This releases the iron into the gas phase where it is singly ionized by the interstellar radiation field. In the extended post-shock region, [FeII] is excited by electron collisions (Mouri et al. 2000), making it a strong diagnostic line for tracing shocks. Since [FeII] is commonly associated with SNRs, the $[\mathrm{FeII}] / \mathrm{Br} \gamma$ line intensity ratio is often used to estimate the abundance of SNRs relative to current star formation. The Shull \& Draine (1987) shock models of $[\mathrm{FeII}] / \mathrm{H} \beta$ imply an [FeII]/Br $\gamma$ ratio of 20-70 (Moorwood \& Oliva 1988), which is in good agreement with SNR observations (Graham et al. 1987; Moorwood et al. 1988). Since the [FeII] emission lifetime of an SNR is approximately $10^{4}$ years (Oliva et al. 1989), the [FeII] luminosity should also be a strong tracer of the SNrate. Qualitatively, the connection between [FeII] emission and supernova activity is supported by a wealth of galactic and extragalactic NIR spectroscopy (Moorwood et al. 1988; Greenhouse et al. 1991; Vanzi \& Rieke 1997) and high-resolution imaging (Forbes et al. 1993; Greenhouse et al. 1997; Alonso-Herrero et al. 2000), which both demonstrate that [FeII] emission often coincides with known radio SNRs and may indeed provide at least a relative measure of SNrate.

However, the quantitative connection between NIR [FeII] emission and supernovae has proved to be more complex, so a better understanding of the underlying physics is necessary. Radio continuum and [FeII] emission do not always correspond, even in a specific SNR. For instance, Greenhouse et al. (1997) find that there is little correlation between the radio continuum and [FeII] line emission in radio-loud SNRs in M82 and suggest that the SNR emission characteristics change as a function of age. In addition, Alonso-Herrero et al. (2003) obtained high-resolution HST [FeII] images of M 82 and NGC 253 and find compact [FeII] emission in only 30-50\% of radio all SNRs. They note that up to $73-78 \%$ of the [FeII] flux is actually diffuse emission and does not originate in the discrete radio SNR, which makes it more difficult to interpret the connection between SNR and [FeII] emission. Vermaas $\&$ van der Werf (in prep.) argue that the absence of [PII] line emission at $1.19 \mu \mathrm{m}$ implies that [FeII] emission is the outcome of grain destruction by strong shocks. This diagnostic allows the 
Table 1. Summary of galaxy parameters.

\begin{tabular}{|c|c|c|c|c|c|c|c|c|}
\hline Galaxy & $\begin{array}{l}\mathrm{RA}^{a} \\
\mathrm{~J} 2000\end{array}$ & $\begin{array}{l}\operatorname{Dec}^{a} \\
\text { J2000 }\end{array}$ & $\begin{array}{c}\text { Morph. }^{b} \\
\text { J2000 }\end{array}$ & Activity $^{c}$ & $\begin{array}{l}\text { Dist. }^{d} \\
{[\mathrm{Mpc}]}\end{array}$ & $\begin{array}{c}c z^{e} \\
{\left[\mathrm{~km} \mathrm{~s}^{-1}\right]}\end{array}$ & $\begin{array}{l}i^{f} \\
{\left[^{\circ}\right]}\end{array}$ & $\begin{array}{c}\log L_{\mathrm{FIR}} g \\
{\left[L_{\odot}\right]}\end{array}$ \\
\hline NGC 3628 & $11 \mathrm{~h} 20 \mathrm{~m} 17.0 \mathrm{~s}$ & $+13 \mathrm{~d} 35 \mathrm{~m} 23 \mathrm{~s}$ & Sb pec edge-on & HII LINER & 12.8 & $847 \pm 2$ & 79.29 & 10.14 \\
\hline NGC 4536 & $12 \mathrm{~h} 34 \mathrm{~m} 27.0 \mathrm{~s}$ & $+02 \mathrm{~d} 11 \mathrm{~m} 17 \mathrm{~s}$ & $\mathrm{SAB}(\mathrm{rs}) \mathrm{bc}$ & HII Sbrst & 15.4 & $1802 \pm 3$ & 58.9 & 10.17 \\
\hline NGC 1792 & $05 \mathrm{~h} 05 \mathrm{~m} 14.4 \mathrm{~s}$ & $-37 \mathrm{~d} 58 \mathrm{~m} 51 \mathrm{~s}$ & $\mathrm{SA}(\mathrm{rs}) \mathrm{bc}$ & HII & 13.2 & $1210 \pm 5$ & 62.78 & 10.22 \\
\hline NGC 1084 & $02 \mathrm{~h} 45 \mathrm{~m} 59.9 \mathrm{~s}$ & $-07 \mathrm{~d} 34 \mathrm{~m} 42 \mathrm{~s}$ & $\mathrm{SA}(\mathrm{s}) \mathrm{c}$ & HII & 16.6 & $1409 \pm 4$ & 46 & 10.42 \\
\hline NGC 1808 & $05 \mathrm{~h} 07 \mathrm{~m} 42.3 \mathrm{~s}$ & $-37 \mathrm{~d} 30 \mathrm{~m} 47 \mathrm{~s}$ & (R)SAB(s)a & HII & 12.3 & $1001 \pm 4$ & 83.87 & 10.55 \\
\hline NGC 520 & $01 \mathrm{~h} 24 \mathrm{~m} 35.1 \mathrm{~s}$ & $+03 \mathrm{~d} 47 \mathrm{~m} 33 \mathrm{~s}$ & pec & Merger Sbrst & 30.5 & $2162 \pm 4$ & 77.49 & 10.81 \\
\hline NGC 7552 & $23 \mathrm{~h} 16 \mathrm{~m} 10.8 \mathrm{~s}$ & $-42 \mathrm{~d} 35 \mathrm{~m} 05 \mathrm{~s}$ & $\left(\mathrm{R}^{\prime}\right) \mathrm{SB}(\mathrm{s}) \mathrm{ab}$ & HII LINER & 22.5 & $1611 \pm 6$ & 23.65 & 11.03 \\
\hline NGC & $23 \mathrm{~h} 22 \mathrm{~m} 00.9 \mathrm{~s}$ & $-42 \mathrm{~d} 28 \mathrm{~m} 50 \mathrm{~s}$ & $\left(\mathrm{R}^{\prime}\right) \mathrm{SB}(\mathrm{s}) 0^{0}$ & HII & 19.3 & $1535 \pm 15$ & 82.44 & $11.43^{h}$ \\
\hline NGC 1614 & $04 \mathrm{~h} 33 \mathrm{~m} 59.8 \mathrm{~s}$ & $-08 \mathrm{~d} 34 \mathrm{~m} 44 \mathrm{~s}$ & $\mathrm{SB}(\mathrm{s}) \mathrm{c}$ pec & Sbrst & 64.2 & $4778 \pm 6$ & 41.79 & 11.43 \\
\hline NGC 6240 & $16 \mathrm{~h} 52 \mathrm{~m} 58.9 \mathrm{~s}$ & $+02 \mathrm{~d} 24 \mathrm{~m} 03 \mathrm{~s}$ & SO-a & LINER & 108.8 & $7242 \pm 45$ & 73.0 & 11.73 \\
\hline Arp 220 & $15 \mathrm{~h} 34 \mathrm{~m} 57.1 \mathrm{~s}$ & $+23 \mathrm{~d} 30 \mathrm{~m} 11 \mathrm{~s}$ & $\mathrm{Sd}$ & LINER Sy2 & 82.9 & $5420 \pm 6$ & 57 & 12.50 \\
\hline
\end{tabular}

Notes. ${ }^{(a)}$ Coordinates of galactic nucleus from NED. ${ }^{(b)}$ NED. ${ }^{(c)}$ Corwin et al. (1994). ${ }^{(d)}$ NED Hubble Flow Distance (Virgo + GA + Shapley where $H_{0}=73.0 \pm 5 \mathrm{~km} \mathrm{~s}^{-1} \mathrm{Mpc}^{-1}$ ) for NGC 520, NGC 7552, NGC 1614, NGC 6240 and Arp 220, metric distances for the rest. ${ }^{(e)}$ Heliocentric radial velocity $(c z)$ from radio measurement from Prugniel et al. (1998) except for NGC 7632 which is from optical measurements. ${ }^{(f)}$ Inclination measured from Prugniel et al. (1998). ${ }^{(g)} L_{\mathrm{FIR}}$ from Sanders et al. (2003). ${ }^{(h)} L_{\mathrm{IR}}$ for NGC 7632 from Yuan et al. (2010).

determination of the excitation mechanism of [FeII]. Vermaas $\&$ van der Werf (in prep.) find in M 83 that, although the [FeII] is diffuse, the distinct lack of [PII] indicates that this emission is nevertheless the consequence of grain destruction by evolved SNRs that have lost their individual identities.

Van der Werf et al. (1993), Calzetti (1997) and Vanzi \& Rieke (1997) have attempted to correlate SNrates to [FeII] emission by finding a relationship between the nonthermal radio continuum and [FeII] flux and using a conversion factor to derive an SNrate. Van der Werf et al. (1993) uses $6 \mathrm{~cm}$ radio fluxes of NGC 6240 from Eales et al. (1990) to estimate SNrates with the conversion factor from Condon \& Yin (1990), which was based on galactic SNR observations. Calzetti (1997) adapted the relation from Van der Werf et al. (1993) to $[\mathrm{FeII}]_{1.26}$. Vanzi \& Rieke (1997) demonstrated that in blue dwarf galaxies, all of the [FeII] emission is plausibly accounted for by SNRs and presented a relationship between [FeII] luminosity and SNrates based on the M 82 calibration. They also found a relationship between [FeII] and age, assuming that most of the [FeII] derives from SNRs.

Thus far, there has been little agreement between the radio continuum and [FeII] line emission in terms of determining SNrates. However, such a relationship is desirable because it allows us to use relatively straightforward NIR [FeII] observations to estimate SNrates whenever individual radio SNRs cannot be resolved. In this paper, we will show that a well determined relationship between [FeII] and SNrate exists, and explore and constrain it in a sample of eleven central regions of nearby starburst galaxies.

First, we will briefly introduce the galaxies in our sample in Sect. 2 and outline the observational parameters in Sect. 3. The results are discussed in Sect. 4, which also displays the spectra, $\mathrm{Br} \gamma, \mathrm{FeII}$, and $\mathrm{H}_{2}$ line-maps and continuum maps. In Sect. 5 we explain the methods used in the analysis, and in Sect. 6 we present our analysis of the [FeII]-SNrate connection. Section 7 explores the dependency of our results on the star formation history of the galaxy and Sect. 8 compares the SNrates derived from the Starburst 99 model to the more classical radio continuum derived SNrates. Finally, our conclusions are highlighted in Sect. 9.

\section{Sample}

We have selected eleven bright nearby starburst galaxies at distances ranging from 10-100 Mpc (see Table 1). All are spiral galaxies with types ranging from $\mathrm{S} 0 \mathrm{a}$ to $\mathrm{Sd}$, with the exception of NGC 520, which is a merging galaxy. Two of the galaxies in our sample are seen edge-on, NGC 520 and NGC 3628. Far-infrared luminosities range from $1.4 \times 10^{10} L_{\odot}$ to $3.2 \times 10^{12} L_{\odot}$, where Arp 220 with $L>10^{12} L_{\odot}$ must be classified as an ultra luminous infrared galaxy (ULIRG), and NGC 6240, NGC 1614 and NGC 7552, all with $L>10^{11} L_{\odot}$ are luminous infrared galaxies (LIRGs). Our sample does not contain galaxies with a dominant active nucleus, but it does have four low ionization nuclear emission line region (LINER) galaxies and one weak Seyfert 2 (Sy2) nucleus.

\subsection{NGC 3628}

NGC 3628 is a nearby $(\sim 12.8 \mathrm{Mpc})$ HII LINER galaxy. It is of morphological type $\mathrm{Sb}$ pec and is seen edge-on. Although this galaxy's nucleus is dominated by a central starburst, it may also be host to a small AGN (González-Martín et al. 2006; Roberts et al. 2001). NGC 3628 is a member of the Leo triplet, a group of 3 closely interacting galaxies. Observations of the HI disk reveal a very disturbed distribution, pointing to the interaction between NGC 3628 and its nearby companion NGC 3627 (Rots 1978; Haynes et al. 1979). Radio observations show an extended radio core of $350 \times 60 \mathrm{pc}$ with a predominately nonthermal spectrum (Hummel 1980; Condon et al. 1982; Reuter et al. 1991). Its inner kiloparsec is dominated by a significant concentration of molecular gas (Israel 2009).

\subsection{NGC 4536}

NGC 4536 is a late type HII/Starburst galaxy that shows $[\mathrm{Ne} \mathrm{V}]$ line emission, which may indicate that the nucleus harbors a weak AGN (Hughes et al. 2005). Previous observations indicate vigorous star formation in the central nuclear region, shown through Br $\gamma$ emission (Puxley et al. 1988). The diffuse radio emission with three central peaks (Vila et al. 1990; Laine et al. 2006) may indicate that this occurs in a ring around the nucleus. There are two ultraluminous X-ray sources, one of which is located in the core of the galaxy and possibly associated with the weak AGN (Liu \& Bregman 2005). 


\subsection{NGC 1792}

NGC 1792 is in a strongly interacting galaxy group with the dominant galaxy NGC 1808. NGC 1792 has surprisingly high luminosities, comparable to those of its more massive partner NGC 1808, at many different wavelengths (Dahlem et al. 1994). Upon closer inspection, Dahlem et al. (1994) found a strongly asymmetric star formation distribution caused by the external trigger of gravitational interactions with NGC 1808.

\subsection{NGC 1084}

NGC 1084 is a HII driven $\mathrm{Sa}(\mathrm{s}) \mathrm{c}$ galaxy located $\sim 16.6 \mathrm{Mpc}$ away. More recently, this galaxy has been classified as an Sbc and $\mathrm{Sb}$ based on its $B$ and $H$ band images, respectively (Eskridge et al. 2002). The $H$ band image reveals a bright nucleus with an elongated bulge. Many weakly defined spiral arms were detected along with many bright knots. NGC 1084 was mapped at $1.49 \mathrm{GHz}$ by Condon (1987), who detected a strong continuum source to the south of the nucleus. Ramya et al. (2007) derived a star formation rate of $2.8 M_{\odot} \mathrm{yr}^{-1}$ along with chaotic star formation that is not necessarily confined to the spiral arms. The northern and southern regions of the galaxy have different star formation rates and typical ages. The north is characterized by a series of short bursts happening intermittently over the past $40 \mathrm{Myr}$, possibly due to an interaction with a gas rich galaxy (Ramya et al. 2007). The southern half of the galaxy is home to much younger stars, <4 Myr (Ramya et al. 2007).

\subsection{NGC 1808}

NGC 1808 is the most massive member of a small group of galaxies. The galactic center has a superwind, compact radio sources, and a molecular ring (Kotilainen et al. 1996). VeronCetty \& Veron (1985) first suspected that NGC 1808 has a faint Seyfert nucleus. This has since been refuted by many (Forbes et al. 1992; Phillips 1993; Krabbe et al. 1994), yet there is recent hard X-ray evidence to support the Seyfert nucleus (Awaki \& Koyama 1993).

\subsection{NGC 520}

Using long slit optical photometry and near-infrared imaging, Stanford \& Balcells (1990) uncovered two separate nuclei in NGC 520, establishing this as a galaxy in the process of merging. Stanford \& Balcells (1991) went on to determine that NGC 520 is most likely the result of a galaxy collision of a gas rich and a gas poor galaxy that happened $\sim 3 \times 10^{8}$ years ago. A thick dust lane at $\mathrm{PA}=95^{\circ}$ completely obscures the primary nucleus at optical wavelengths, but the secondary nucleus is visible to the northwest of the primary. There are strong plumes of ionised gas, thought to represent a bipolar outflow away from the starburst dominated nucleus (Norman et al. 1996).

\subsection{NGC 7552}

NGC 7552 is a LIRG HII galaxy and host to a weak LINER. This galaxy has a starburst ring surrounding the nucleus. It does not appear to be overly disturbed, which suggests a slow bar mediated star formation evolution. Due to the quiescent nature of the nucleus, NGC 7552 provides a near perfect environment to study the bar and starburst ring around it. In addition to the bar, there are two dominant spiral arms and two weaker rings at radii of 1.9 and $3.4 \mathrm{kpc}$ (Feinstein et al. 1990). Forbes et al. (1994b) found an inner ring with a radius of $1 \mathrm{kpc}$ in the radio, which is not visible in the NIR continuum but is visible in NIR color maps and $\mathrm{Br} \gamma$ emission. The dominant circumnuclear ring is at a distance of $850 \mathrm{pc}$ from the center of the nucleus and the spiral arms provide a flow of molecular gas into this region (Schinnerer et al. 1997).

\subsection{NGC 7632}

NGC 7632 is a member of a loose galaxy group, containing multiple galaxies. This is a ring galaxy, which is observed to be distorted and bent towards the other group members (Arp 1981). The interacting galaxies located south of the NGC 7632 also appear strongly distorted with much absorbing material (Arp 1981).

\subsection{NGC 1614}

NGC 1614 is a SB(s)c peculiar LIRG with both LINER and starburst activity. Although only one NIR peak has been observed, Neff et al. (1990) considered the tidal tails or plumes to be evidence that NGC 1614 is the result of a merger of at least two galaxies. A central nucleus of $45 \mathrm{pc}$ is surrounded by a $600 \mathrm{pc}$ diameter ring of current star formation (Alonso-Herrero et al. 2001). The Bry emission reveals a double peaked morphology (Kotilainen et al. 2001), in agreement with the radio continuum (Condon et al. 1982). Further studies of NGC 1614 by Olsson et al. (2010) found the molecular gas distribution double peaked at $R=300 \mathrm{pc}$ with an additional weaker peak in the center of the nucleus. A radio continuum ring is also found at $R=300 \mathrm{pc}$ which is triple peaked, each consistent with the brightness temperature of SNR. Olsson et al. (2010) concludes that the LINER spectrum is due to shocks associated with supernovae and therefore most likely caused by star formation and not AGN activity.

\subsection{NGC 6240}

NGC 6240 just falls short of being a traditional ULIRG with a luminosity of $L_{\mathrm{IR}}=10^{12} L_{\odot}$ but otherwise has all the characteristics of the class. The extended tidal tails seen in the optical indicate a merger. NGC 6240 hosts a double nucleus separated by $1.5^{\prime \prime} \pm 0.1^{\prime \prime}$ as seen in ground based observations in the optical (Schulz et al. 1993) and near-infrared (Doyon et al. 1994), yet a separation of $2^{\prime \prime} \pm 0.15^{\prime \prime}$ as measured in $[\mathrm{FeII}]_{1.64} \mu \mathrm{m}$ (Van der Werf et al. 1993) and radio observations (Carral et al. 1990; Eales et al. 1990; Beswick et al. 2001). The high infrared luminosity is partially due to the powerful nuclear starburst and partially to an AGN continuum (Genzel et al. 1998).

\subsection{1. $\operatorname{Arp} 220$}

The ULIRG Arp 220 emits $99 \%$ of its bolometric luminosity in the infrared (Soifer et al. 1984; Emerson et al. 1984). Arp 220 is a very gas rich galaxy, with the highest density component in the nucleus, with a molecular gas density up to $\sim 10^{-8} \mathrm{~cm}^{-3}$ (Rangwala et al. 2011). This high IR luminosity and intense starburst activity points to a merger, supported by the existence of two nuclei, separated by $\sim 1^{\prime \prime}$ (Scoville et al. 1997). Arp 220 has an extremely high extinction, with estimates running from $A_{\mathrm{v}} \sim 50$ to 1000 (Sturm et al. 1996; Downes \& Solomon 1998). Smith et al. (1998) resolve the radio nucleus of Arp 220 and find many radio point sources, which they identify as SNRs. They 
deduce a star formation rate of $50-100 M_{\odot} \mathrm{yr}^{-1}$ and a corresponding SNrate of $1.75-3.5 \mathrm{yr}^{-1}$.

\section{Observations}

Observations of the sample galaxies were made with the Spectrograph for INtegral Field Observations in the NearInfrared (SINFONI) at the VLT. SINFONI provides spatial and spectral data in the form of data cubes in $J, H$, and $K$ bands. The SINFONI instrument is mounted at the Cassegrain focus of the Unit Telescope 4 at the Very Large Telescope (VLT).

We took observations in all three $J, H$, and $K$ bands using a spatial pixel scale of $0.25^{\prime \prime}$ corresponding to a field of view of $8^{\prime \prime} \times 8^{\prime \prime}$ and a spectral resolution of 2000, 3000 and 4000 respectively, between October, 2006 and March, 2007. All science observations were taken in the $\mathrm{ABA}^{\prime}$ nodding mode ( $300 \mathrm{~s}$ of object $300 \mathrm{~s}$ of sky $300 \mathrm{~s}$ of object), where $A^{\prime}$ is slightly offset from $A$. The object exposures are averaged during the reconstruction of the data cube.

We extracted additional observations from the SINFONI archive (http://archive.eso.org/wdb/wdb/eso/sinfoni/ form). These observations include five galaxies, Arp 220, NGC 1614, NGC 1808, NGC 6240 and NGC 7552. As selection criteria we required the galaxies to be bright and nearby, and to have archival observations in the $0.25^{\prime \prime}$ spatial resolution mode in all three bands and $300 \mathrm{~s}$ integration times.

We used the standard reduction techniques of the SINFONI pipeline on all observations. including corrections for flat field, dark current, nonlinearity of pixels, distortion, and wavelength calibration. We obtained the flux calibration and atmospheric corrections from observations of a standard star. Finally, we calibrated the continuum fluxes to match the published 2MASS observations in the same aperture, and we determined a "flux correction factor" for each galaxy in each observed band, and we applied these correction factors to the line intensities as well.

\section{Results}

\subsection{Spectra}

We show the spectra of each galaxy center integrated over a high signal-to-noise region in the $J$ (Fig. 1), $H$ (Fig. 2) and $K$ bands (Fig. 3). The actual spatial area over which the spectra were integrated is overplotted in the $K$ band images in Figs. 4 and 5. We have focused on non-nuclear regions to illustrate the spectral signature of the extended emission. The dominant emission lines in each band are marked with dotted lines, notably $\mathrm{FeII}_{1.26}$ and $\mathrm{Pa} \beta$ in the $J$ band, $\mathrm{FeII}_{1.64}$ in the $H$ band and $\mathrm{Br} \gamma, \mathrm{HeI}_{2.06}$, and multiple $\mathrm{H}_{2}$ lines in the $K$ band.

\subsection{Continuum and linemaps}

The SINFONI datacubes allow us to construct $J, H$ and $K$ continuum maps as well as linemaps for each of the emission lines detected in these bands. These emission lines provide important diagnostics to trace specific physical processes. The $\operatorname{Pa} \beta(1.282 \mu \mathrm{m})$ and $\operatorname{Br} \gamma(2.166 \mu \mathrm{m}) \mathrm{HI}$ lines trace massive, young star formation. The [FeII] emission lines, which emit most strongly at 1.257 and $1.644 \mu \mathrm{m}$, are commonly used as tracers of strong shocks associated with supernova remnants, nuclear winds, or jets. There is also a wealth of ro-vibrational $\mathrm{H}_{2}$ lines throughout the $H$, and $K$ bands, the brightest of which occur at rest wavelengths of $2.122 \mu \mathrm{m}$ (1-0 S(1)), $2.248 \mu \mathrm{m}(2-1 \mathrm{~S}(1))$, and $2.034 \mu \mathrm{m}(1-0 \mathrm{~S}(2)) . \mathrm{H}_{2}$ can be excited by UV florescence from massive stars, or thermally by shocks from supernovae or stellar winds. The relative intensities of the various $\mathrm{H}_{2}$ lines indicates which of these physical processes is exciting the gas.

In Figs. 4 and 5 we present the $K$ band continuum, $\operatorname{Br} \gamma$, [FeII $]_{1.26}$ and $\mathrm{H}_{2,2.12}$ line maps for each of the galaxies in our sample. The continuum maps were created by finding the average continuum level at each pixel, excluding any emission lines. The linemaps were created using QFitsView and DPUSER's evaluated velocity map function "evalvelmap", developed by the Max Planck Institute for Extraterrestrial Physics and available at http://www . mpe.mpg.de/ ott/QFitsView/. This function fits a gaussian profile to the emission line at each pixel.

The first galaxy in Fig. 4 is the edge-on NGC 3628, which has a strong central dust lane running from east to west. This is best seen by comparing the $\mathrm{Br} \gamma$ and $[\mathrm{FeII}]$ maps. Since $K$-band $\mathrm{Br} \gamma$ emission is at a longer wavelength, it is less prone to extinction and the morphology resembles that of the $K$ band continuum, with an additional emission peak at the western edge of the galaxy. The $J$-band [FeII] emission is at a shorter wavelength and is more affected by the dust lane. It shows an asymmetric hour glass morphology, with the brightest regions above the $K$ band nucleus. The $\mathrm{H}_{2}$ emission is similar to the $\mathrm{Br} \gamma$ emission, but it has more diffuse morphology with strong emission throughout the very central region.

NGC 4536 reveals a star forming ring best seen in $\operatorname{Br} \gamma$ and [FeII] emission. The $\mathrm{H}_{2}$ emission is concentrated at the $K$ band continuum peak, with extended diffuse emission throughout the ring. Both $\mathrm{Br} \gamma$ and $[\mathrm{FeII}]$ peak in the northwest corner of the galaxy but their peaks do not coincide.

NGC 1792 has a $K$-band continuum morphology similar to that of NGC 4536, but the line emission is much weaker in this galaxy. The low signal-to-noise ratios prevent us from identifying any real structure. However, in the $\operatorname{Br} \gamma$ map a small peak occurs at the $K$ band continuum maximum, with structures suggesting spiral arms emanating from the nucleus. Although both $\mathrm{H}_{2}$ and [FeII] emission peaks at the end of these "arms", the actual [FeII] fluxes hardly exceed the noise level. The $\mathrm{H}_{2}$ has a higher signal-to-noise than the other emission lines and there are is distinct $\mathrm{H}_{2}$ peaks emission in the nuclear region.

In NGC 1084, all three emission lines appear to peak on the $K$-band continuum maximum. The $\mathrm{Br} \gamma$ and $\mathrm{H}_{2}$ maps show a somewhat similar distribution, with extended diffuse emission along the major axis line, and a weak secondary peak to the north. The [FeII] map shows a single central maximum surrounded by weak diffuse emission.

NGC 1808 also has a star-formation ring, weakly seen in both the $\mathrm{Br} \gamma$ and [FeII] maps and a few local maxima also seen in the $\mathrm{H}_{2}$ map. The ring appears to be very asymmetric, with relative bright fluxes at the eastern edge and almost no emission from the western edge. The $\mathrm{H}_{2}$ emission is distributed more symmetrically around the $K$ band peak.

In Fig. 5, we first examine NGC 520, another nearly edgeon galaxy that also hosts a dense dust lane running east to west. The $\mathrm{Br} \gamma$ morphology is very asymmetric, peaking to the west of the $K$-band continuum maximum. The $\mathrm{H}_{2}$ emission peaks at the same location as the $\mathrm{Br} \gamma$, but has a secondary peak east of the $K$ band center. The [FeII] emission suffers from extinction by the dust lane and only weak [FeII] emission is seen to the southwest of the galaxy mid plane.

NGC 7552 is a face-on galaxy with a very clear starformation ring around the nucleus. The $\mathrm{Br} \gamma$ emission shows a pronounced minimum in the center, but clear individual maxima in the ring. Unlike Forbes et al. (1994a), who found the $\mathrm{H}_{2}$ morphology to match that of the $K$ band continuum, we find the 


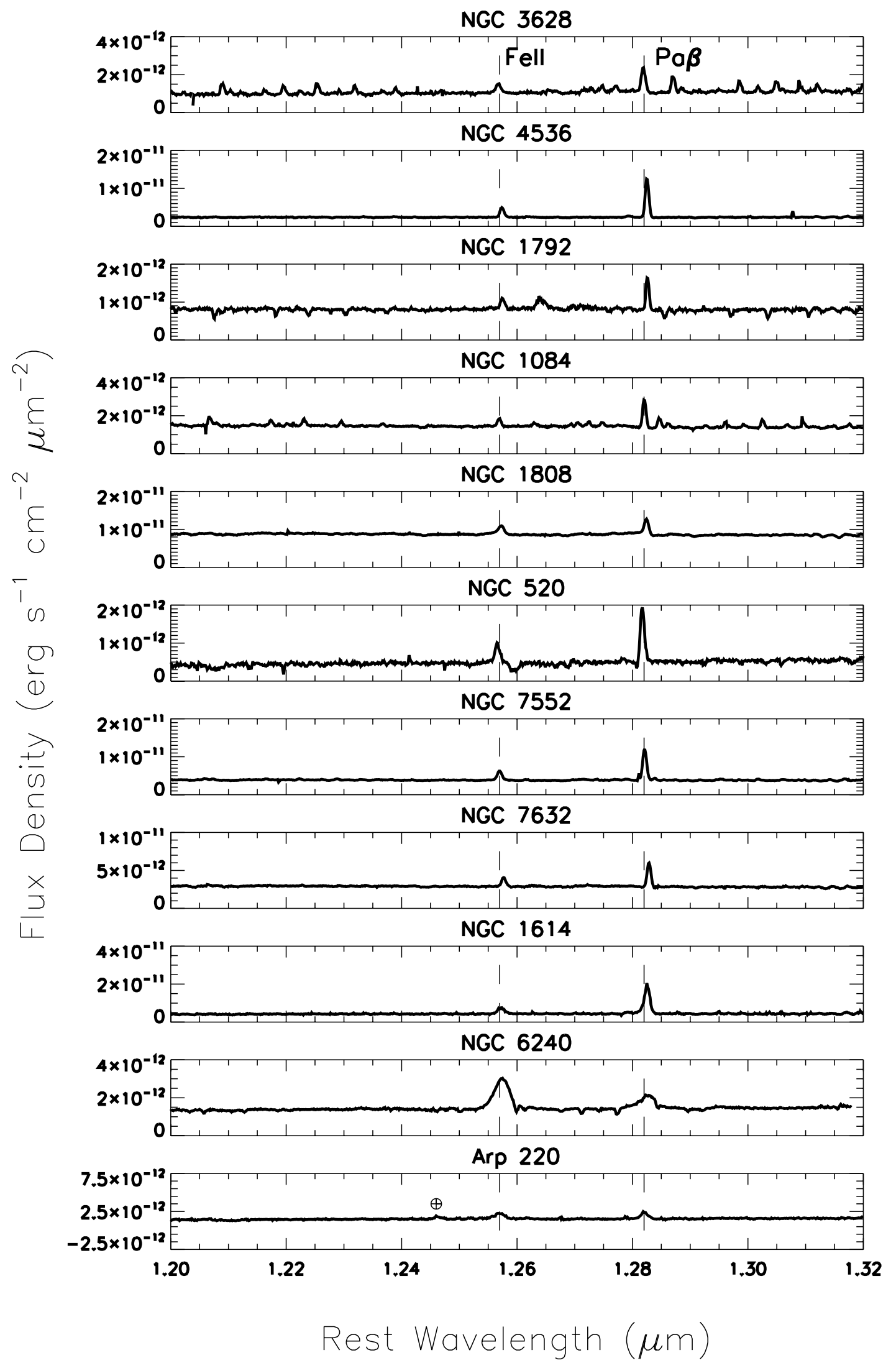

Fig. 1. Spatially integrated rest frame spectra from the nucleus of each galaxy in the $J$ band. The spatial area used in the integration is shown in Figs. 4 and 5 by a black rectangle. Each spectrum is integrated over an $8 \times 8$ pixel area. $\mathrm{FeII}_{1.26}$ and $\mathrm{Pa} \beta$ emission lines are denoted by dashed lines and marked along the top of the figure. The broad feature near $1.25 \mu \mathrm{m}$ in the Arp 220 spectra is an atmospheric artifact along with the narrow absorption features in NGC 520, and the numerous broad peaks between 1.2-1.24 and 1.29-1.32 $\mu \mathrm{m}$ in the NGC 3628, NGC 1792 and NGC 1084 spectra. 


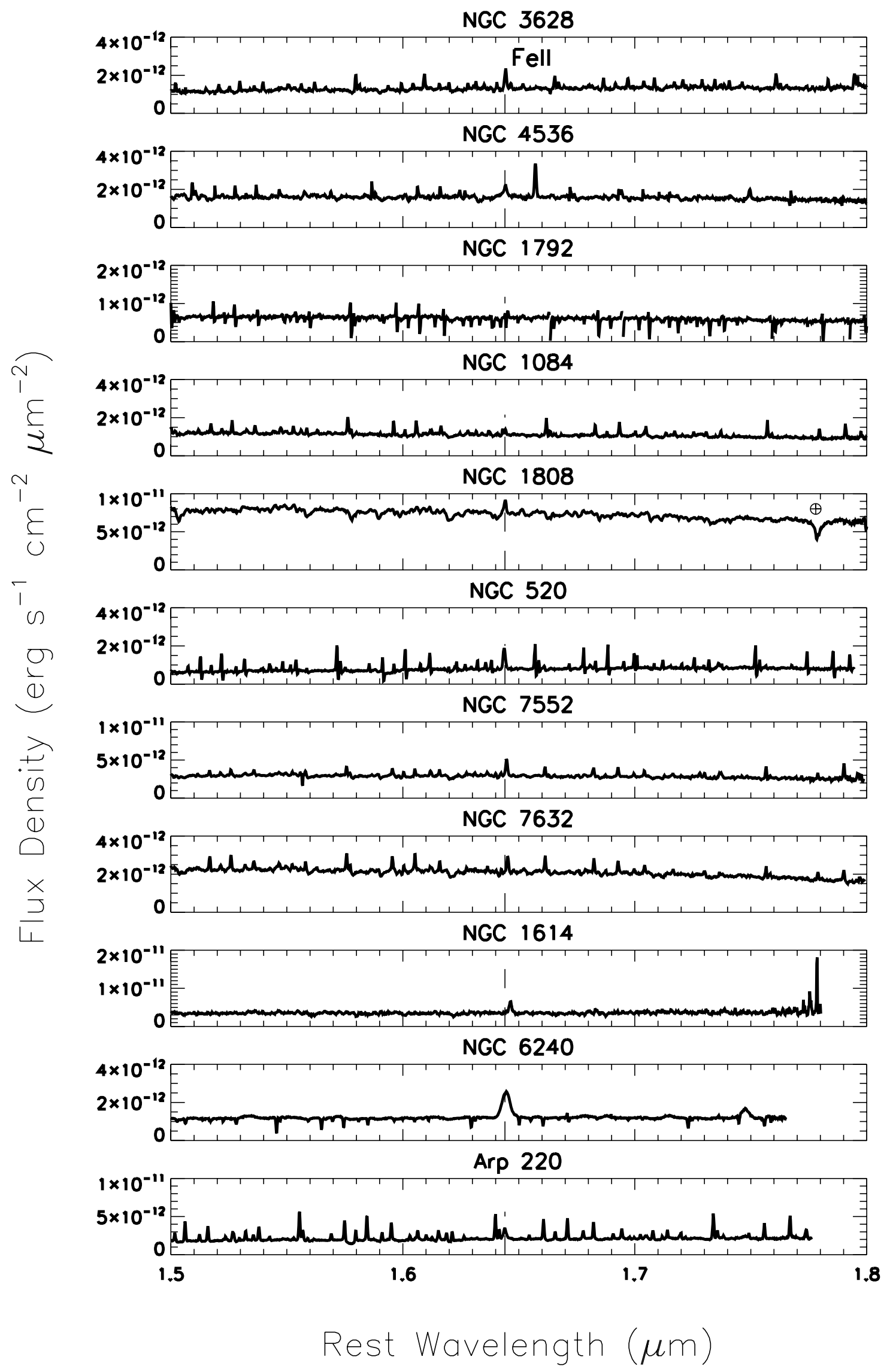

Fig. 2. Spatially integrated rest frame spectra from the nucleus of each galaxy in the $H$ band. The spatial area used in the integration is shown in Figs. 4 and 5 by a black rectangle. Each spectrum is integrated over an $8 \times 8$ pixel area. The FeII ${ }_{1.64}$ emission line is denoted by a dashed line and marked along the top of the figure. Several spectra are degraded by residual atmospheric $\mathrm{OH}$ features. 
M. J. F. Rosenberg et al.: [FeII] as a tracer of supernova rate

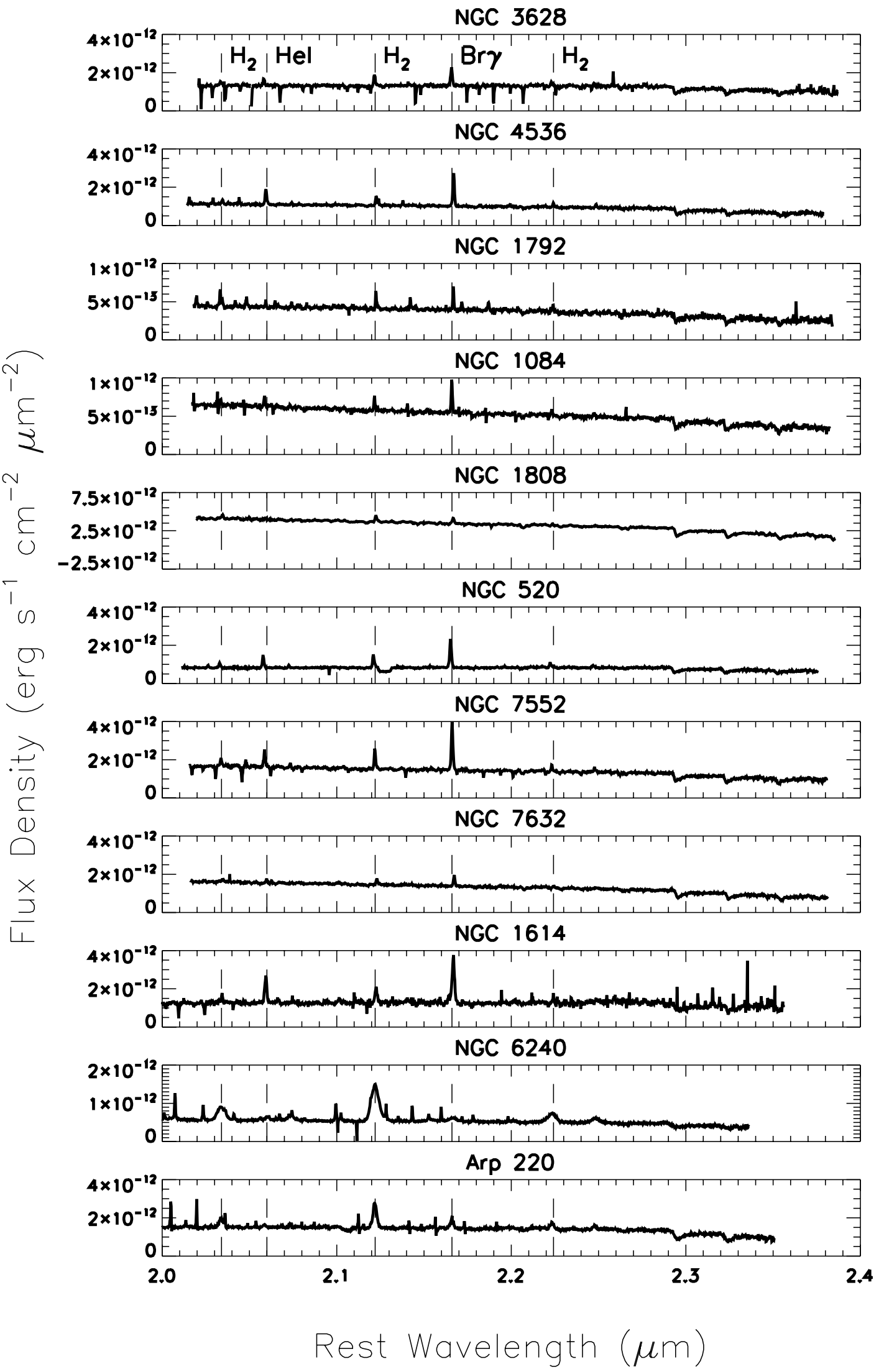

Fig. 3. Spatially integrated rest frame spectra from each galaxy in the $K$ band. The spatial area used in the integration is shown in Figs. 4 and 5 by a black rectangle. Each spectrum is integrated over an $8 \times 8$ pixel area. $\mathrm{Br} \gamma, \mathrm{H}_{2}$, and $\mathrm{HeI}_{2.06}$ emission lines are denoted by dashed lines and marked along the top of the figure. Residual atmospheric contamination is present in the form of narrow peaks in the spectra of NGC 1614 and Arp 220 and as sharp absorption features in the spectra of NGC 3628 and NGC 7552. 

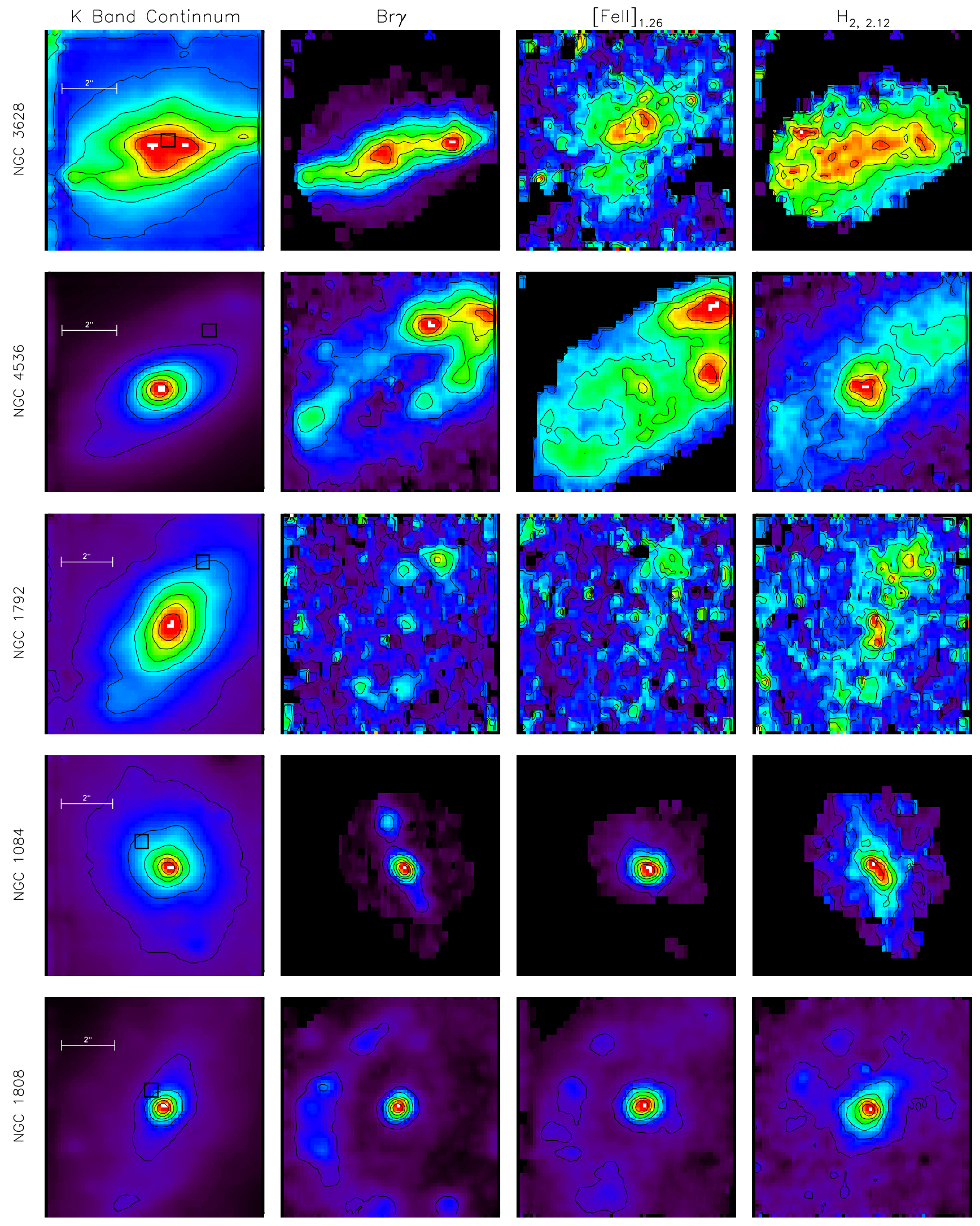

Fig. 4. $K$ band continuum map, $\mathrm{Br} \gamma$, $[\mathrm{FeII}]_{1.26}$, and $\mathrm{H}_{2,2.12}$ line maps of NGC 7632, NGC 3628, NGC 4536, NGC 1792, and NGC 1084. The pixel scale is given by the $2^{\prime \prime}$ scale bar in the $K$ band column. The black rectangle marks the areas over which the spectra in Figs. 1-3 were integrated. In all figures, north is up. 
M. J. F. Rosenberg et al.: [FeII] as a tracer of supernova rate
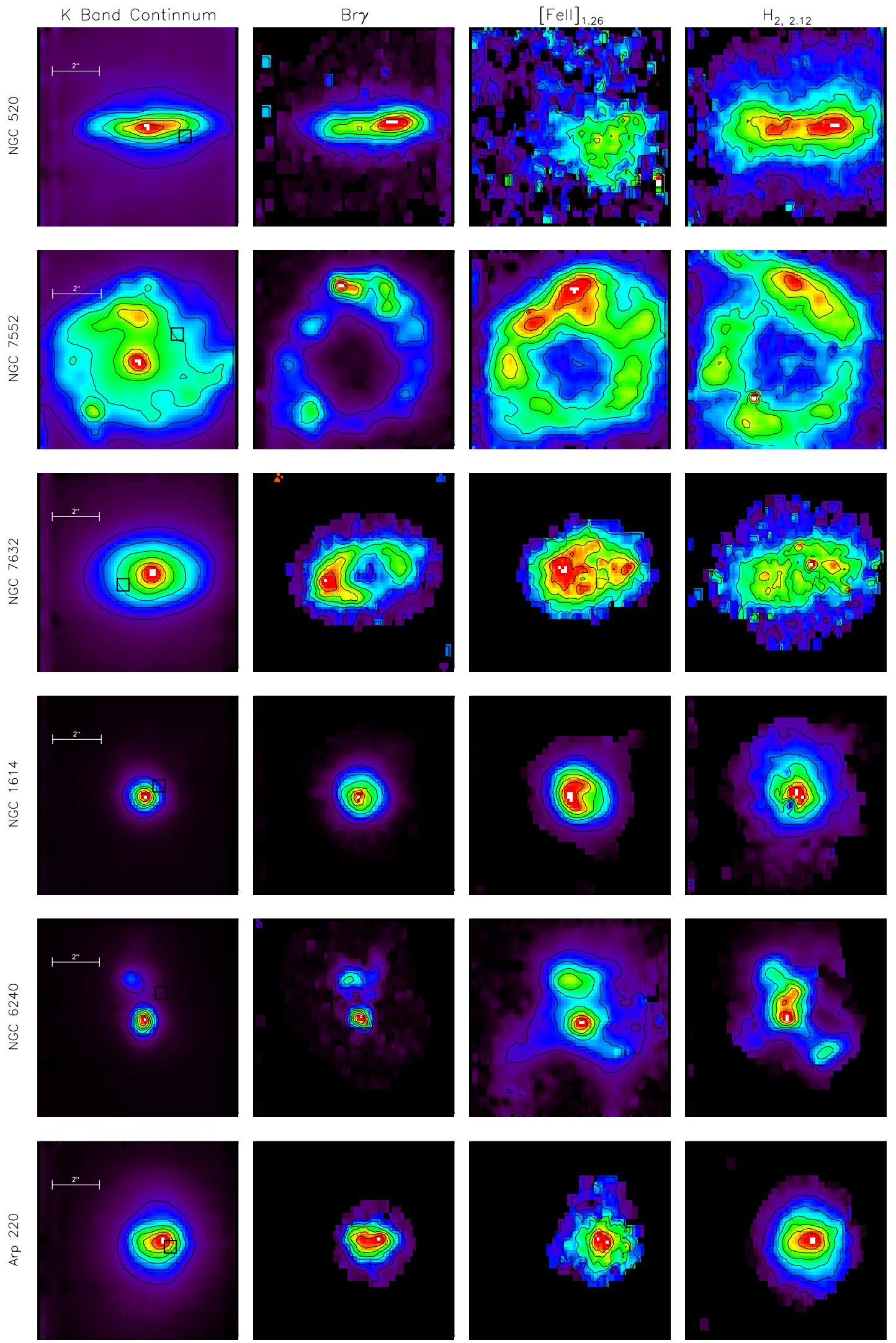

Fig. 5. $K$ band continuum map, $\mathrm{Br} \gamma$, $[\mathrm{FeII}]_{1.26}$, and $\mathrm{H}_{2,2.12}$ line maps of NGC 1808, NGC 520, NGC 7552, NGC 1614 , NGC 6240 and Arp 220. The pixel scale is given by the $2^{\prime \prime}$ scale bar in the $K$ band column. The black rectangle represents the area over which the spectra in Figs. 1-3 were integrated. In all figures, north is up. 

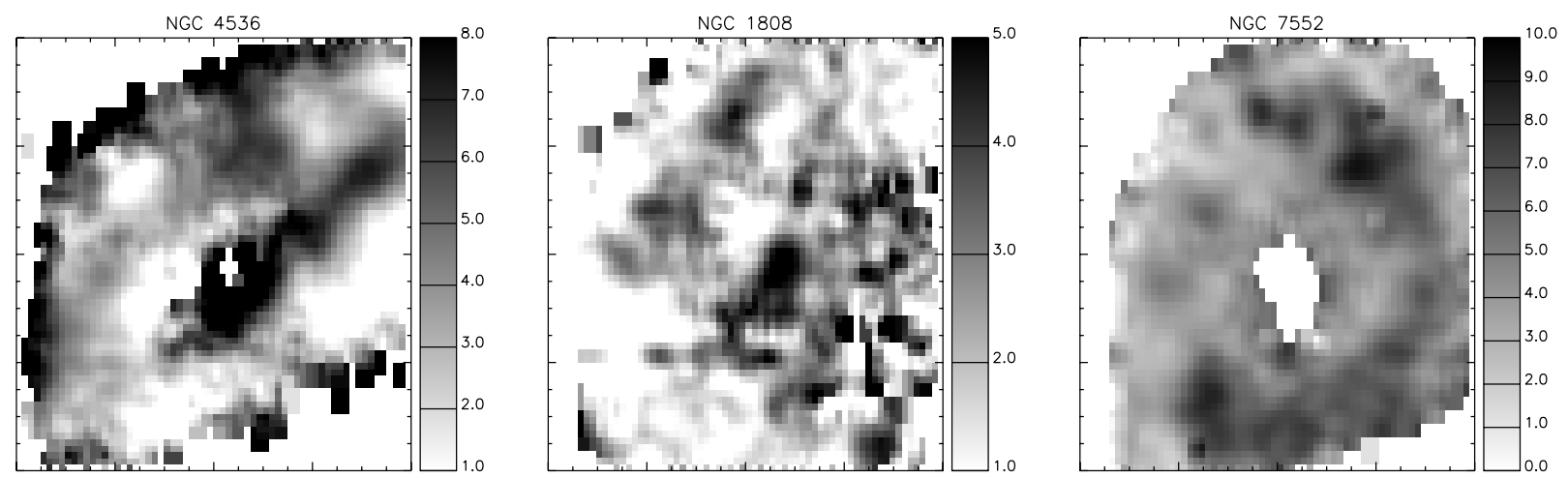

Fig. 6. Extinction maps of NGC 4536, NGC 1808 and NGC 7552. The color scales are displayed to the right of each map, the values represent $A_{\mathrm{V}}$ measured in magnitudes.

distributions of the $\mathrm{H}_{2}$ and the $\mathrm{Br} \gamma$ emission to be very similar, with an arm or bar-like structure extending from the northern $\mathrm{Br} \gamma$ peaks. The $[\mathrm{FeII}]$ emission is different: it shows roughly the same ring-like shape, but with emission peaks in very different positions.

NGC 7632 reveals an asymmetric ring in $\mathrm{Br} \gamma$ emission. The emission is much stronger on the east side of the galaxy in both $\mathrm{Br} \gamma$ and $[\mathrm{FeII}]$ emission. The east side is in the direction of the other group members (Arp 1981). The distortion and asymmetry is most likely evidence of the tidal interaction with its partner galaxies.

NGC 1614 has a very compact and radially symmetric morphology in the $K$ band, $\mathrm{Br} \gamma$ and $\mathrm{H}_{2}$ emission lines. However, the [FeII] emission shows a crescent morphology around the nucleus.

The double nucleus of NGC 6240 is evident in the $K$ band continuum and the $\mathrm{Br} \gamma$ line images. Less clearly, the double nucleus also shows up in the $\mathrm{H}_{2}$ and [FeII] images that reveal a more complex morphology. In all images, strongest emission coincides with southern nucleus.

Arp 220 is another compact galaxy that appears fairly symmetric in the $K$ band and all the emission line maps. The peak is slightly off center towards the western side of the galaxy and there are two separate peaks in the $[\mathrm{FeII}]$ emission.

\section{Methods}

To further study the correlation between [FeII] emission and SNrate, we have performed a pixel by pixel analysis. We evaluated the continuum flux, emission line strength and equivalent width at each pixel in the SINFONI datacubes in order to gain insight into the variation of physical properties (such as ages and excitation mechanisms) across each galactic nucleus. We first corrected the linemaps for extinction, and used the $\mathrm{Br} \gamma$ equivalent widths as an input into Starburst 99 to calculate SNrates. These could then be correlated with extinction corrected [FeII] luminosities.

\subsection{Extinction correction}

In order to study the intrinsic emission line strengths, it is necessary to determine the degree to which dust obscures each nucleus. We accomplish this by identifying emission lines with fixed intrinsic intensity ratios, and comparing the observed to the intrinsic line ratio. In our NIR database, the best line pair to use is $\mathrm{Pa} \beta$ ( $\mathrm{H} \mathrm{5-3)}$ and $\mathrm{Br} \gamma(\mathrm{H} \mathrm{7-4)}$, which has a ratio of 5.88 (Hummer \& Storey 1987) over a wide range of physical conditions. Extinction is also probed by the $[\mathrm{FeII}]_{1.26} /[\mathrm{FeII}]_{1.64}$ ratio, in which both [FeII] lines originate in the same upper level with an intrinsic ratio of 1.36 (Nussbaumer \& Storey 1988). For completeness sake, we calculated both line ratios and compared the extinction maps. There is little disparity in morphology between the [FeII] derived and HI dervied extinction maps. It is clear, however, that the [FeII] lines are of limited use, as the spectral baseline they define is small and provides relatively little differential extinction. More importantly, the [FeII] lines have lower signal-to-noise ratios than the $\mathrm{J}$ and $\mathrm{K}$ lines, the $[\mathrm{FeII}]_{1.64}$ being particularly bad in this respect as it is degraded by residual atmospheric $\mathrm{OH}$ contamination in many of our observations. As a result, meaningful extinction maps based on the [FeII] line ratio could only be derived over limited areas for a minority of the galaixes. Thus, the extinctions derived from the $\mathrm{Pa} \beta / \mathrm{Br} \gamma$ ratio should be the more reliable and will be used to complete the following analysis.

We calculated the extinction at each pixel and created an extinction map revealing the regions most effected by dust. Extinction maps for three example galaxies are shown in Fig. 6. Table 2 lists the averaged visual extinction $\left(A_{\mathrm{V}}\right)$ over the observed field of view for both line ratios. The table also includes the integrated, extinction corrected $\mathrm{Br} \gamma, \mathrm{Pa} \beta$, and [FeII] line fluxes. The maps were integrated over the full field of view but were filtered for low signal-to-noise pixels and pixels with linewidths either too narrow or too broad. The visual extinction $A_{\mathrm{V}}$ is determined assuming using the near-infrared extinction law $A_{\lambda} \propto \lambda^{-1.8}$ (Martin \& Whittet 1990). The disparity between the extinctions derived from the $\mathrm{Fe}$ and $\mathrm{H}$ lines mostly reflects the poor quality of the former, as discussed above. In the case of NGC 1792, the little line emission observed has a very low signal-to-noise ratio (cf. Fig. 4), negatively affecting the accuracy of any $A_{\mathrm{V}}$ measurement, hence also the accuracy of the extinction corrected [FeII] and $\mathrm{Br} \gamma$ fluxes. In NGC 6240, the extremely low $\mathrm{Br} \gamma$ flux makes it difficult to accurately determine the extinction on a pixel by pixel basis.

In order to obtain the best possible result, we constructed extinctio corrected $[\mathrm{FeII}]_{1.26}$ and $\mathrm{Br} \gamma$ line maps using pixel-bypixel extinctions derived from the $\mathrm{Pa} \beta / \mathrm{Br} \gamma$ line ratio only. We also constructed pixel-by-pixel maps of the $\mathrm{Br} \gamma$ equivalent width directly from the observed $\mathrm{Br} \gamma$; these are therefore independent of the assumed extinction. The $\operatorname{Br} \gamma$ equivalent width and the Br $\gamma$ luminosity thus determined are the only observational inputs used in the calculation of SNrates from the Starburst 99 model, described in detail in Sect. 5.2 below. 
Table 2. Unextinction corrected $\mathrm{Br} \gamma, \mathrm{Pa} \beta$, $[\mathrm{FeII}]_{1.26}$, and $[\mathrm{FeII}]_{1.64}$ fluxes with the $A_{\mathrm{V}}$ derived by the $\mathrm{Pa} \beta / \mathrm{Br} \gamma$ intrinsic line ratio.

\begin{tabular}{lccccc}
\hline \hline Galaxy & $\begin{array}{c}\mathrm{Pa} \beta \\
\left(\mathrm{erg} \mathrm{s}^{-1} \mathrm{~cm}^{-2}\right)\end{array}$ & $\begin{array}{c}\mathrm{Br} \gamma \\
10^{-14}\left(\mathrm{erg} \mathrm{s}^{-1} \mathrm{~cm}^{-2}\right)\end{array}$ & $\begin{array}{c}{[\mathrm{FeII}]_{1.26}} \\
10^{-14}\left(\mathrm{erg} \mathrm{s}^{-1} \mathrm{~cm}^{-2}\right)\end{array}$ & $\begin{array}{c}{[\mathrm{FeII}]_{1.64}} \\
10^{-14}\left(\mathrm{erg} \mathrm{s}^{-1} \mathrm{~cm}^{-2}\right)\end{array}$ & $\begin{array}{c}A_{\mathrm{V} \mathrm{Br} \gamma} a \\
(\mathrm{mag})\end{array}$ \\
\hline NGC 3628 & 0.7 & 1.4 & 1.4 & 0.6 & 20.3 \\
NGC 4536 & 12.7 & 3.5 & 3.4 & 4.5 & 3.9 \\
NGC 1792 & 1.3 & 0.3 & 0.1 & - & 2.9 \\
NGC 1084 & 1.0 & 0.3 & 0.3 & 0.5 & 3.0 \\
NGC 1808 & 18.3 & 4.4 & 9.2 & 8.0 & 2.7 \\
NGC 520 & 1.2 & 1.3 & 1.2 & 0.3 & 15.3 \\
NGC 7552 & 20.7 & 6.8 & 6.3 & 8.1 & 5.3 \\
NGC 7632 & 1.8 & 0.5 & 0.5 & 0.8 & 3.9 \\
NGC 1614 & 13.8 & 3.5 & 3.1 & 3.7 & 3.3 \\
NGC 6240 & 3.3 & 0.6 & 8.5 & 8.7 & 15.6 \\
Arp 220 & 0.4 & 0.5 & 0.7 & - & 15.4 \\
\hline
\end{tabular}

Notes. All measurements have a 10\% error, which is the estimated calibration uncertainty. NGC 1792 has a $20 \%$ error due to the low signal to noise of this observation. ${ }^{(a)} A_{\mathrm{V}}$ is determined assuming using the near-infrared extinction law $A_{\lambda} \propto \lambda^{-1.8}$ (Martin \& Whittet 1990). Although the measured $A_{\mathrm{V}}$ is very high in some galaxies, the extinction measured in the NIR wavelength range is significantly less.

\subsection{Calculating SNrate}

Starburst 99 (from here on referred to as SB99) is a tool that models spectrophotometric properties of star-forming galaxies (Leitherer et al. 1999) such as spectral energy distributions (SEDs), luminosities, equivalent widths, supernova rates and colors. This includes predictions of the variations in these properties as a function of starburst age. The models have been calculated for 5 different metallicities $(Z=$ $0.04,0.02,0.008,0.004,0.001)$ and 3 different initial mass functions (IMF). In addition, the two extreme star-formation modes are considered: the continuous mode in which star formation proceeds continuously at a constant rate, and the instantaneous mode where it has the form of a delta function starburst.

In our analysis, we assumed a near solar metallicity $(Z=$ $0.02)$, a Salpeter IMF $\left(\alpha=2.35, M_{\text {low }}=1 M_{\odot}, M_{\text {up }}=100 M_{\odot}\right)$ and an instantaneous star formation mode. In the case of an instantaneous starburst, the model normalizes the burst to an initial starburst mass of $10^{6} M_{\odot}$. We calculated the average age of the population dominating the emission in each pixel from the observed $\mathrm{Br} \gamma$ equivalent width $(\mathrm{EW}(\mathrm{Br} \gamma))$, which is independent of this normalization. SB99 provides the expected SNrate as a function of the age thus determined. Due to the normalisation of $10^{6} M_{\odot}$, the SNrate must be appropriately scaled by comparing the SB99 age dependent prediction of ionising photon flux $\left(\mathrm{N}\left(\mathrm{H}^{\circ}\right)\right)$ to the observed ionising photon flux, which in turn scales with $\mathrm{Br} \gamma$ luminosity. This comparison yields a scaling factor that is directly proportional to the initial mass and initial star formation rate. This allowed us to determine the actual SNrates based on the true initial conditions of the region represented in each pixel.

\section{Analysis}

In this section, we use the observed linemaps and the SB99 results to do a detailed analysis of each galaxy in order to better understand the complex relationship between [FeII] emission and SNrate. First, we present the age and SNrate estimates for each galaxy. Then we will provide both a qualitative and quantitative comparison of the SNrates and the [FeII] luminosity.

\subsection{Age and SNrate}

Table 3 lists the Br $\gamma$ equivalent widths as well as the average age and integrated SNrate using the SB99 instantaneous burst model
Table 3. Average equivalent width of $\operatorname{Br} \gamma$ and the SB99 derived average ages and integrated SNrates using the instantaneous starburst model.

\begin{tabular}{lccc}
\hline \hline Galaxy & $\begin{array}{c}E W(\mathrm{Br} \gamma) \\
\AA\end{array}$ & $\begin{array}{c}\mathrm{Age}_{\text {inst }} \\
(\mathrm{Myr})\end{array}$ & $\begin{array}{c}\mathrm{SNrate}_{\text {inst }} \\
\mathrm{yr}^{-1}\end{array}$ \\
\hline NGC 7632 & 5.9 & 7.0 & 0.03 \\
NGC 3628 & 7.5 & 6.9 & 0.01 \\
NGC 4536 & 10.6 & 6.7 & 0.3 \\
NGC 1792 & 4.1 & 7.9 & 0.007 \\
NGC 1084 & 4.8 & 7.3 & 0.009 \\
NGC 1808 & 7.2 & 6.9 & 0.06 \\
NGC 520 & 11.7 & 6.6 & 0.3 \\
NGC 7552 & 13.7 & 6.7 & 0.3 \\
NGC 7632 & 5.9 & 7.0 & 0.03 \\
NGC 1614 & 22.9 & 6.4 & 0.9 \\
NGC 6240 & 3.6 & 7.7 & 3.6 \\
Arp 220 & 8.0 & 6.8 & 0.7 \\
\hline
\end{tabular}

for each galaxy. Although the analysis was done on a pixel-topixel basis, the values given in Table 3 are either averaged (in the case of age and equivalent width) or integrated (in the case of the SNrate) over the galaxy.

\subsection{Qualitative correlation}

To illustrate the morphological relation between the [FeII] emission and the SNrate, we show a side-by-side comparison of the $K$-band continuum, the extinction-corrected $\mathrm{Br} \gamma$ flux, the extinction corrected $[\mathrm{FeII}]_{1.26}$ flux, and the derived SNrate in Fig. 7 for the galaxies NGC 4536, NGC 1808, and NGC 7552, which have the highest quality observations. A visual comparison of the morphologies in the four different maps of each galaxy shows that the SNrate map most closely resembles that of the [FeII] emission.

For instance, in NGC 4536 there is a bright knot of Br $\gamma$ emission directly north of the nucleus with secondary emission peaks to the northwest and southeast of the galaxy. The [FeII] emission is concentrated on the galaxy nucleus with a long plume of emission (perhaps an inner spiral arm) extending from the nucleus towards the northwest. A small knot of faint [FeII] emission coincides with the Br $\gamma$ peak. Mimicking the [FeII] emission, the SNrate peaks at the center and also shows an arm extending towards the northwest. The SNrate exhibits a slight increase at the $\mathrm{Br} \gamma$ peak, but on the whole more closely resembles the morphology of [FeII] emission. 

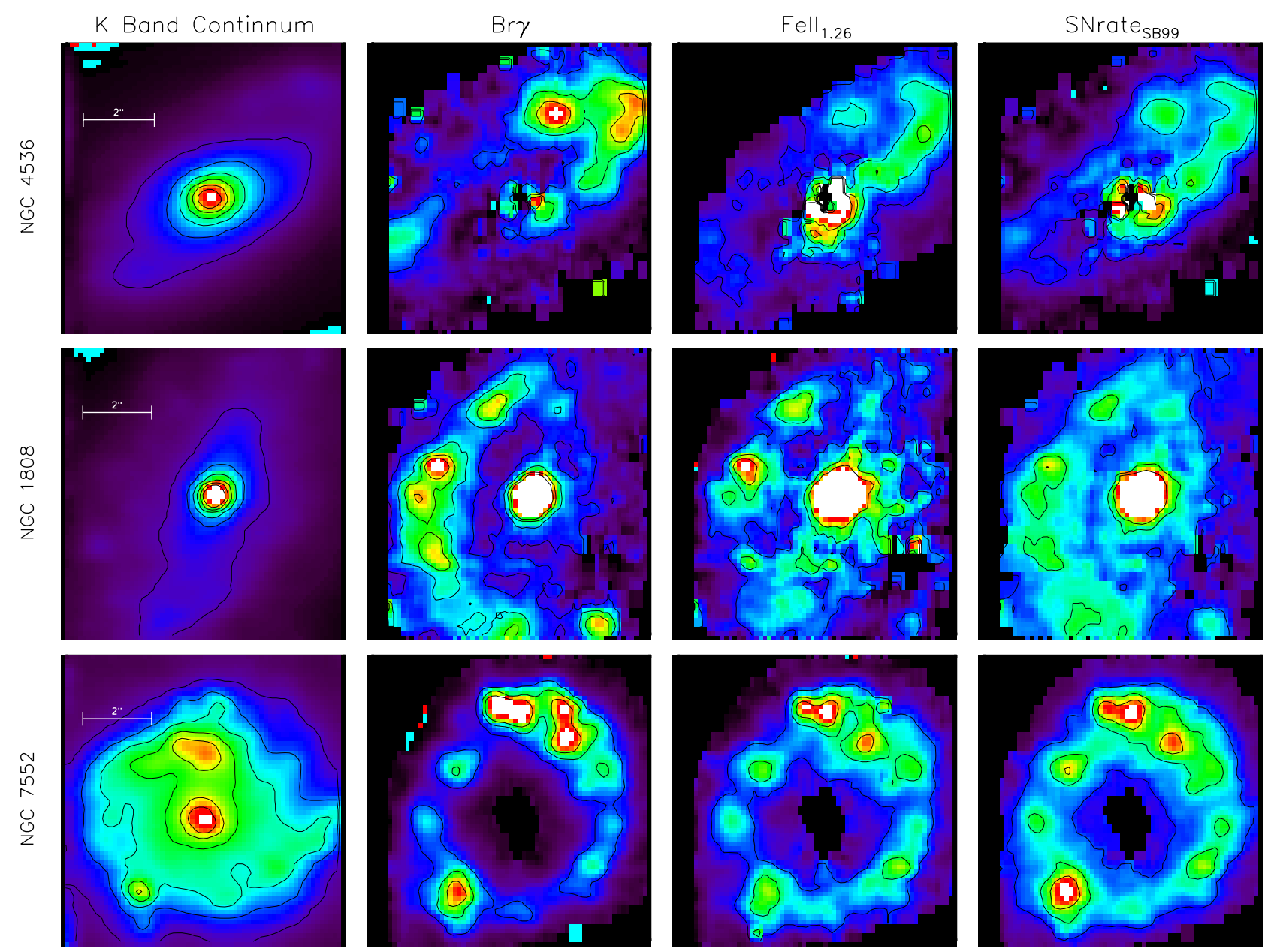

Fig. 7. A comparison between the $K$-band continuum, Br $\gamma$ line map, $[\mathrm{Fe} I \mathrm{II}]_{1.26}$ line map, and the SNrate morphologies for NGC 4536, NGC 1808, and NGC 7552. The black discontinuities represent areas that have been filtered due to low signal-to-noise values in the line or extinction maps. The white pixels represent the highest flux regions.

The starburst ring surrounding the bright nucleus of NGC 1808 shows up clearly in Br $\gamma$. The ring contains individual bright knots of $\mathrm{Br} \gamma$ emission. The ring is much less prominent in [FeII] emission. The SNrate map shows a ring structure more clear than in the [FeII] map but it is quite diffuse and lacks the contrast that the $\mathrm{Br} \gamma$ knots provide. The SNrate map also shows diffuse extended structure around the nucleus, similar to the $[\mathrm{FeII}]$ emission but distinct from the $\mathrm{Br} \gamma$ map in which the compact nucleus is much more isolated.

Finally, the face-on spiral, NGC 7552, also reveals its starburst ring in $\mathrm{Br} \gamma$, with little emission at the nucleus. In the northern part of the ring we find three major $\operatorname{Br} \gamma$ knots, two slightly northwest of the nucleus and one elongated knot directly north of the nucleus. Comparison shows that only one of the two northwestern $\mathrm{Br} \gamma$ knots is discernible in [FeII] emission. We also see that the morphology in the elongated knot differs in the two maps. Focusing on the SNrate morphology, the northern emission knots resemble the relative flux and morphologies of the [FeII] emission much more closely than the $\mathrm{Br} \gamma$ emission. Specifically, the top northwestern knot is also missing in SNrate map and the elongated northern $\mathrm{Br} \gamma$ peak is resolved to two peaks, resembling the relative flux ratios seen in [FeII].

All comparisons show that, at least qualitatively, the SNrate maps resemble the $[\mathrm{FeII}]$ maps much more than those of the $\mathrm{Br} \gamma$ emission on a pixel by pixel basis. Once again we emphasise that the only observational input into the SNrate calculation is the $\mathrm{Br} \gamma$ luminosity and $\mathrm{EW}(\mathrm{Br} \gamma)$; the [FeII] flux is never used. As the SNrate nevertheless correlates better with the $[\mathrm{FeII}]$ than with the $\operatorname{Br} \gamma$ emission, we conclude that this strongly supports $[\mathrm{FeII}]$ as a robust tracer of SNrate.

\subsection{Quantitative correlation}

Now that we have established that a qualitative correlation exists between [FeII] emission and SNrates, it is important to verify to what extent this relationship holds up quantitatively. To do this, we compare in Fig. 8 for each galaxy the $[\mathrm{Fe} I I]_{1.26}$ luminosity and the SNrate derived from Bry pixel-by-pixel. The linemaps were not additionally filtered, with the exception of NGC 6240 which was filtered to eliminate pixels where the $\mathrm{Br} \gamma$ flux is insufficient to determine the true extinction. We normalised both the SNrate and the [FeII] luminosity to values per square parsec so that each pixel represents the same physical area. We performed a least squares regression in log space on the combined points from all sample galaxies except NGC 1792 because of its poor signal-to-noise data. The combined linear regression is indicated by a solid black line in Fig. 8; it takes the analytical form:

$\log \frac{v_{\mathrm{SNrate}}}{\mathrm{yr}^{-1} \mathrm{pc}^{-2}}=(1.01 \pm 0.2) * \log \frac{[\mathrm{FeII}]_{1.26}}{\mathrm{erg} \mathrm{s}^{-1} \mathrm{pc}^{-2}}-41.17 \pm 0.9$.

The errors represent the standard deviation of the slopes and intercepts of the individual galaxies, thus representing the 


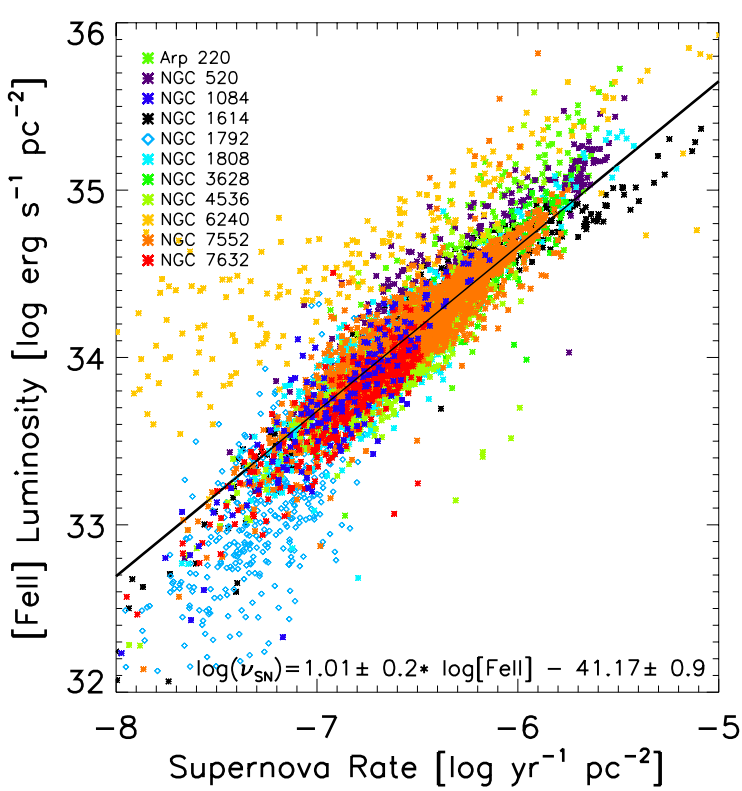

Fig. 8. A pixel-pixel plot of SNrate, as derived from SB99, compared to [FeII] luminosity. Each galaxy is represented with a different color and the values are normalised to a square parsec. The black line represents the best fit power law excluding NGC 1792, which is represented by diamonds.

variation between individual galaxies' fits. This is an intrinsic error, corresponding to the variations in properties among the galaxies and not derived from the quality of the observation. To investigate the strength of the relationship on a global basis, we have plotted, excluding NGC 1792, the integrated SNrate plotted against the integrated [FeII] luminosity for each galaxy, again with the best fit regression line. Errors in the extinction are a potentially significant source of uncertainty in the derived relationship. The dashed lines at either side of this best fit in Fig. 9 mark the regression lines we would find if the extinction magnitudes were overestimated respectively underestimated by factors of two. It is obvious from the figure that our result is essentially unaffected by this uncertainty. The best fit for the global values is given by:

$\log \frac{v_{\mathrm{SNrate}}}{\mathrm{yr}^{-1} \mathrm{pc}^{-2}}=(0.89 \pm 0.2) * \log \frac{[\mathrm{FeII}]_{1.26}}{\mathrm{erg} \mathrm{s}^{-1} \mathrm{pc}^{-2}}-36.19 \pm 0.9$

Thus, the averaged and the integrated SNrate - [FeII] luminosity relation are identical within the errors. Both procedures yield a power law with a slope of nearly unity. The relation is linear well within the errors.

An important source of uncertainty in the SNrate calculation is caused by observational errors in the $\mathrm{Br} \gamma,[\mathrm{FeII}]$ and $K$ band continuum intensities. These observational errors are dominated by the uncertainty in the calibration, which is of the order of $10 \%$ of the flux in all cases except NGC 1792, where the errors are dominated by noise, estimated to be about $20 \%$ of the flux. The uncertainties in the derived SNrate are the same as those in $\mathrm{Br} \gamma$ flux, i.e. about $10 \%$. However, the observational errors are not the dominant source of uncertainty. This is the systematic error introduced by the choice of the starburst model. Because we use SB99, our choices are limited to two star-formation models only, instantaneous and continuous. In the next section, we discuss the effects of the choice of the burst model on the relation between SNrate and [FeII] luminosity.

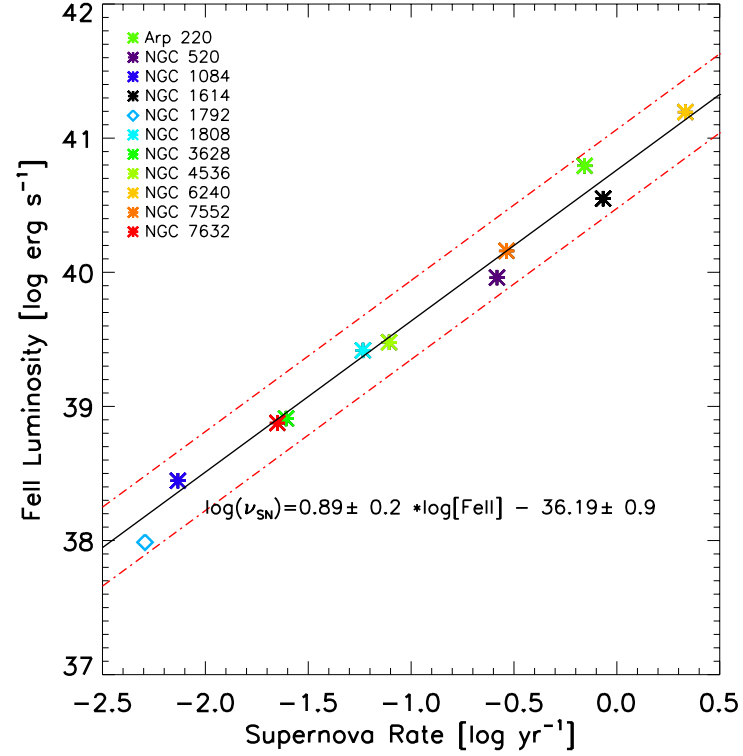

Fig. 9. Integrated SNrate plotted against integrated [FeII] luminosity over the field of view. The black line is the least squares regression line, excluding NGC 1792. The red dotted lines represent the least squares regression line if the magnitude of extinction was overestimated respectively underestimated by a factor of two in optical depth.

\section{Dependency on burst model}

So far, we have based our analysis on the assumption that star formation in the sample galaxies is best represented by an instantaneous burst. The other extreme case also modelled by SB99 is the continuous star-formation scenario. It is unlikely that either of these extreme cases is a true representation of the situation in the galaxies considered; we suspect that reality is somewhere in between and better described by one or more star bursts extended in time. We cannot determine the actual star formation history of the sample, but the SB99 model allows us to constrain the validity of our determination of SNrate/[FeII] luminosity relation by performing the same analysis this time assuming a continuous star-formation mode. The result is shown in (Fig. 10) as a plot of the [FeII] luminosity versus the SNrate for the combined pixels of all sample galaxies. In this figure, the black dots represent the SNrates assuming an instantaneous burst (as in Fig. 8) and the red points represent the same pixels where the SNrate is calculated assuming continuous star formation.

From Fig. 10 it is clear that also in continuous star-formation mode, the SNrate is still closely related to the [FeII] luminosity. However, in this case, the relation is no longer linear as the best fit slope is 1.20 . We also note that in this situation, a given [FeII] luminosity also implies an SNrate roughly an order of magnitude higher than in the instantaneous burst model.

\section{Comparison to radio SNrate}

Which mode best represents our sample? Radio continuum emission is the classical tracer of SNrate, and radio flux densities can be used to obtain an independent estimate of the SNrate for comparison to the SNrates derived with the SB99 models. We apply the equation given by Huang et al. (1994), which relates nonthermal radio luminosity and SNrate, to VLA observations. There are no VLA archived observation of NGC 7632 and this galaxy is excluded from the comparison. A similar situation applies to NGC 7552, but here we could use the ATCA 


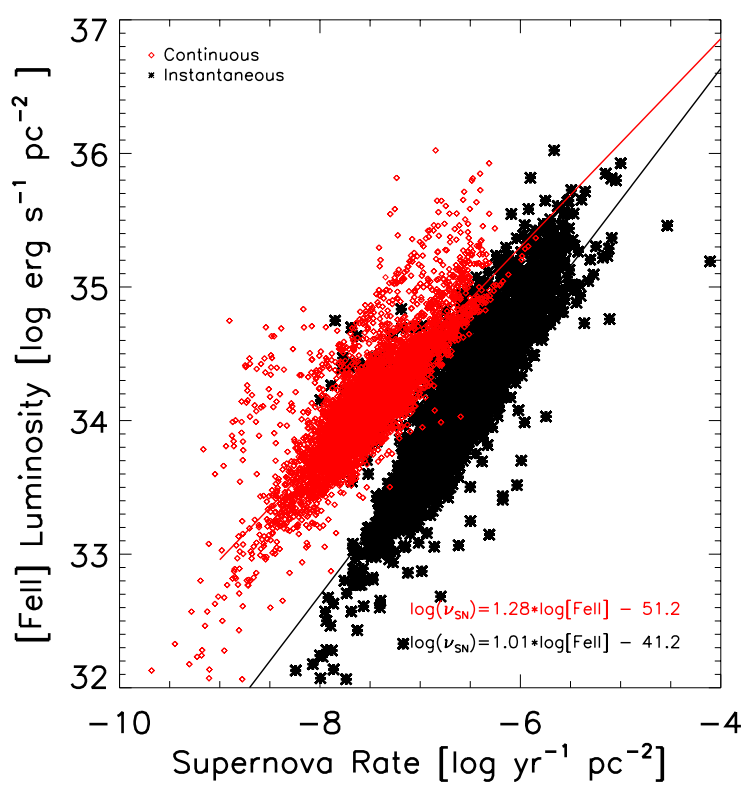

Fig. 10. Pixel-pixel comparison of SNrate to [FeII] luminosity. The black points represent the pixel values for all the galaxies using the instantaneous burst model, while the red points represent the same pixels using the continuous burst model.

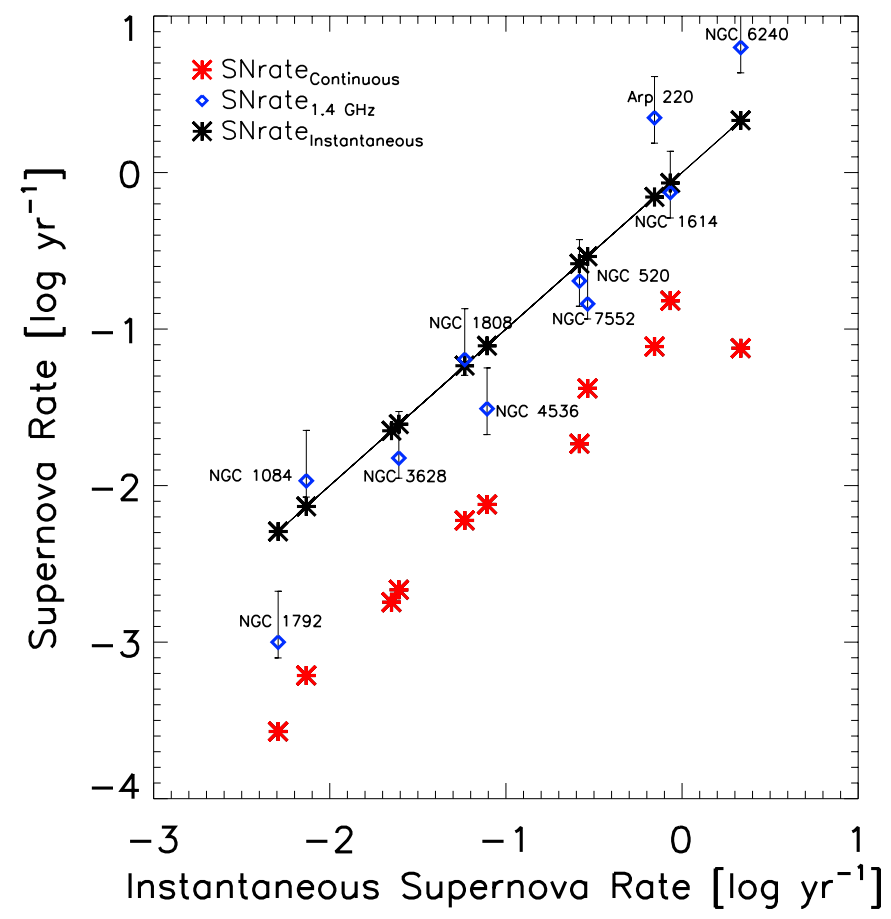

Fig. 11. Comparison of the SB99 instantaneous SNrate ( $x$-axis) to the radio SNrate (blue diamond), the SB99 continuous SNrate (red asterisk) and for comparison the SB99 instantaneous SNrate (black asterisk).

4.8 GHz map instead. For the nearest extended galaxies, we matched the radio integration area to that of SINFONI. For the more distant NGC 1614, NGC 6240, and Arp 220, we used NED VLA $1.4 \mathrm{GHz}$ integrated flux densities since these galaxies are compact enough that the entire galaxy is encompassed in the SINFONI field of view. In Fig. 11 we compare the radio SNrates thus derived to the SB99 instantaneous and continuous SNrates.

For all galaxies, except NGC 1792, NGC 4536, NGC 6240 and Arp 220, the radio SNrate closely matches those given by the SB99 instantaneous burst model. As noted before, the NIR emission linemaps of NGC 1792 are too noisy to be reliable. However, NGC 4536 is a nearly face-on galaxy with relatively low extinction and high signal-to-noise spectra, and the NIR data should be reliable. Thus, NGC 4536 is undergoing star formation that is closer to instantaneous but extended in time. Arp 220 and NGC 6240 have radio SNrates even higher than the SB99 instantaneous SNrates. Also, in Fig. 8, the points for these galaxies lie systematically above the best fit line, with a similar slope to the rest of the galaxies. This offset can be explained by either an excess of [FeII] emission or a deficiency of $\mathrm{Br} \gamma$ emission, since these are the two observables that are used to scale SNrate and [FeII] luminosity. In the case of Br $\gamma$ deficiency, the Br $\gamma$ emission could be selectively suppressed by dust absorption of Lyman continuum photons in these very dense systems. However, in NGC 6240 and Arp 220, it is tempting to speculate that this excess is caused by merger related shocks that are over exciting the [FeII] in addition to the SNRs. Evidence for large scale shocks in NGC 6240 comes from the prominent wings on the $\mathrm{H}_{2} 1-0$ $\mathrm{S}$ (1) line which trace the shocked superwind (Van der Werf et al. 1993; Engel et al. 2010). The [FeII] emission is morphologically similar to the $\mathrm{H}_{2}$, indicating that the [FeII] also traces the superwind. These powerful merger (U)LIRGs may demonstrate a limit to the validity of the direct relationship between SNrate and [FeII] luminosity. In addition, determining an accurate extinction in (U)LIRGs presents a challenge. The under or over estimation of extinction leads to inaccurate [FeII] luminosities as well as Br $\gamma$ luminosities used to scale the SNrate. However, the offset between the (U)LIRGs and the other galaxies is minor. They share a common slope and appear to be offset only in [FeII] emission. The validity of this relationship in the case of (U)LIRGs can only be established by studying a larger sample of these galaxies. In any case, it appears that the majority of (modest) starburst galaxies is well represented by the assumption of a (nearly) instantaneous burst of star formation. In addition, the very good agreement between SNrates derived from the radio continuum, and from NIR data provides added confidence in the diagnostic strength of [FeII] as a tracer of SNrate.

\section{Conclusion}

Using SINFONI observations of 11 nearby galaxies, we have performed a pixel-by-pixel analysis of the correlation between $[\mathrm{FeII}]_{1.26}$ emission and independently derived SNrates. We measured accurate $\mathrm{Br} \gamma, \mathrm{Pa} \beta,[\mathrm{FeII}]_{1.26},[\mathrm{FeII}]_{1.64}$, and $\mathrm{H}_{2,2.12}$ line fluxes. We determined $\mathrm{Br} \gamma$ equivalent widths which were used as input into the SB99 model to find the starburst age in each pixel. In the SB99 model, this defines an SNrate, normalised to an initial mass of $10^{6} M_{\odot}$, which we scaled with the observed $\mathrm{Br} \gamma$ luminosity to find the actual SNrate in each pixel.

The comparison of the $[\mathrm{FeII}]_{1.26}$ luminosity to the SNrate (derived only from $\mathrm{Br} \gamma$ equivalent width and luminosity) reveals a nearly linear correlation, where the error represents the spread of intrinsic properties over our sample of galaxies,

$\log \frac{v_{\mathrm{SNrate}}}{\mathrm{yr}^{-1} \mathrm{pc}^{-2}}=1.01 \pm 0.2 * \log \frac{[\mathrm{FeII}]_{1.26}}{\mathrm{erg} \mathrm{s}^{-1} \mathrm{pc}^{-2}}-41.17 \pm 0.9$.

This relationship is valid both on a pixel-pixel basis and for the integrated galaxy. For the integrated [FeII] luminosity and SNrate, the fit is remarkably tight with very little spread. However, to correctly determine the absolute SNrates, it is still critical to determine whether star formation has occurred in a (nearly) instantaneous burst, or has proceeded in a continuous fashion. SNrates derived from radio continuum observations 
may be used to distinguish these scenarios. Most of the modest starburst galaxies in our sample are best fitted assuming instantaneous star formation. However, we find that the relationship breaks down for (U)LIRGs in our sample.

From the strong correlations found in this sample of galaxies, we confidently conclude that $[\mathrm{FeII}]_{1.26}$ emission line strengths are quantitatively correlated with SNrates and can be used to derive these rates either locally or globally. This use of [FeII] as a robust tracer of SNrate provides us with a very useful diagnostic tool. It is particularly important as it allows determination of SNrates from NIR observations of distant galaxies, where individual SNRs can not be resolved.

\section{References}

Alonso-Herrero, A., Rieke, G. H., Rieke, M. J., \& Scoville, N. Z. 2000, ApJ, 532,845

Alonso-Herrero, A., Engelbracht, C. W., Rieke, M. J., Rieke, G. H., \& Quillen, A. C. 2001, ApJ, 546, 952

Alonso-Herrero, A., Rieke, G. H., Rieke, M. J., \& Kelly, D. M. 2003, AJ, 125, 1210

Arp, H. 1981, ApJS, 46, 75

Awaki, H., \& Koyama, K. 1993, Adv. Space Res., 13, 221

Beswick, R. J., Pedlar, A., Mundell, C. G., \& Gallimore, J. F. 2001, MNRAS, 325,151

Calzetti, D. 1997, AJ, 113, 162

Carral, P., Turner, J. L., \& Ho, P. T. P. 1990, ApJ, 362, 434

Condon, J. J. 1987, ApJS, 65, 485

Condon, J. J. 1992, ARA\&A, 30, 575

Condon, J. J., \& Yin, Q. F. 1990, ApJ, 357, 97

Condon, J. J., Condon, M. A., Gisler, G., \& Puschell, J. J. 1982, ApJ, 252, 102

Corwin, Jr., H. G., Buta, R. J., \& de Vaucouleurs, G. 1994, AJ, 108, 2128

Dahlem, M., Bomans, D. J., \& Will, J. 1994, ApJ, 432, 590

Downes, D., \& Solomon, P. M. 1998, ApJ, 507, 615

Doyon, R., Wells, M., Wright, G. S., et al. 1994, ApJ, 437, L23

Eales, S. A., Becklin, E. E., Hodapp, K.-W., Simons, D. A., \& Wynn-Williams, C. G. 1990, ApJ, 365, 478

Emerson, J. P., Clegg, P. E., Gee, G., et al. 1984, Nature, 311, 237

Engel, H., Davies, R. I., Genzel, R., et al. 2010, A\&A, 524, A56

Eskridge, P. B., Frogel, J. A., Pogge, R. W., et al. 2002, ApJS, 143, 73

Feinstein, C., Mendez, M., Vega, I., \& Forte, J. C. 1990, A\&A, 239, 90

Forbes, D. A., Boisson, C., \& Ward, M. J. 1992, MNRAS, 259, 293

Forbes, D. A., Ward, M. J., Rotaciuc, V., et al. 1993, ApJ, 406, L11

Forbes, D. A., Kotilainen, J. K., \& Moorwood, A. F. M. 1994a, ApJ, 433, L13

Forbes, D. A., Norris, R. P., Williger, G. M., \& Smith, R. C. 1994b, AJ, 107, 984

Genzel, R., Lutz, D., Sturm, E., et al. 1998, ApJ, 498, 579

González-Martín, O., Masegosa, J., Márquez, I., Guerrero, M. A., \& DultzinHacyan, D. 2006, A\&A, 460, 45

Graham, J. R., Wright, G. S., \& Longmore, A. J. 1987, ApJ, 313, 847

Graham, J. R., Wright, G. S., \& Longmore, A. J. 1990, ApJ, 352, 172

Greenhouse, M. A., Woodward, C. E., Thronson, Jr., H. A., et al. 1991, ApJ, 383,

164
Greenhouse, M. A., Satyapal, S., Woodward, C. E., et al. 1997, ApJ, 485, 438

Haynes, M. P., Giovanelli, R., \& Roberts, M. S. 1979, ApJ, 229, 83

Huang, Z. P., Thuan, T. X., Chevalier, R. A., Condon, J. J., \& Yin, Q. F. 1994, ApJ, 424, 114

Hughes, M. A., Axon, D., Atkinson, J., et al. 2005, AJ, 130, 73

Hummel, E. 1980, A\&AS, 41, 151

Hummer, D. G., \& Storey, P. J. 1987, MNRAS, 224, 801

Israel, F. P. 2009, A\&A, 506, 689

Kotilainen, J. K., Forbes, D. A., Moorwood, A. F. M., Van der Werf, P. P., \& Ward, M. J. 1996, A\&A, 313, 771

Kotilainen, J. K., Reunanen, J., Laine, S., \& Ryder, S. D. 2001, A\&A, 366, 439

Krabbe, A., Sternberg, A., \& Genzel, R. 1994, ApJ, 425, 72

Laine, S., Kotilainen, J. K., Reunanen, J., Ryder, S. D., \& Beck, R. 2006, AJ, 131,701

Leitherer, C., Schaerer, D., Goldader, J. D., et al. 1999, ApJS, 123, 3

Liu, J., \& Bregman, J. N. 2005, ApJS, 157, 59

Martin, P. G., \& Whittet, D. C. B. 1990, ApJ, 357, 113

Moorwood, A. F. M., \& Oliva, E. 1988, A\&A, 203, 278

Moorwood, A. F. M., Oliva, E., \& Danziger, I. J. 1988, in Supernova Remnants and the Interstellar Medium, ed. R. S. Roger, \& T. L. Landecker, IAU Colloq., 101,391

Mouri, H., Kawara, K., \& Taniguchi, Y. 2000, ApJ, 528, 186

Neff, S. G., Hutchings, J. B., Standord, S. A., \& Unger, S. W. 1990, AJ, 99, 1088

Norman, C. A., Bowen, D. V., Heckman, T., Blades, C., \& Danly, L. 1996, ApJ, 472, 73

Nussbaumer, H., \& Storey, P. J. 1988, A\&A, 193, 327

Oliva, E., Moorwood, A. F. M., \& Danziger, I. J. 1989, A\&A, 214, 307

Oliva, E., Moorwood, A. F. M., \& Danziger, I. J. 1990, A\&A, 240, 453

Olsson, E., Aalto, S., Thomasson, M., \& Beswick, R. 2010, A\&A, 513, A11

Phillips, A. C. 1993, AJ, 105, 486

Prugniel, P., Zasov, A., Busarello, G., \& Simien, F. 1998, A\&AS, 127, 117

Puxley, P. J., Hawarden, T. G., \& Mountain, C. M. 1988, MNRAS, 234, 29P

Ramya, S., Sahu, D. K., \& Prabhu, T. P. 2007, MNRAS, 381, 511

Rangwala, N., Maloney, P. R., Glenn, J., et al. 2011, ApJ, 743, 94

Reuter, H., Krause, M., Wielebinski, R., \& Lesch, H. 1991, A\&A, 248, 12

Roberts, T. P., Schurch, N. J., \& Warwick, R. S. 2001, MNRAS, 324, 737

Rots, A. H. 1978, AJ, 83, 219

Sanders, D. B., Mazzarella, J. M., Kim, D.-C., Surace, J. A., \& Soifer, B. T. 2003, AJ, 126, 1607

Schinnerer, E., Eckart, A., Quirrenbach, A., et al. 1997, ApJ, 488, 174

Schulz, H., Fried, J. W., Röser, S., \& Keel, W. C. 1993, A\&A, 277, 416

Scoville, N. Z., Yun, M. S., \& Bryant, P. M. 1997, ApJ, 484, 702

Shull, J. M., \& Draine, B. T. 1987, in Interstellar Processes, ed. D. J. Hollenbach, \& H. A. Thronson Jr., Astrophys. Space Sci. Libr., 134, 283

Smith, H. E., Lonsdale, C. J., Lonsdale, C. J., \& Diamond, P. J. 1998, ApJ, 493, L17

Soifer, B. T., Neugebauer, G., Helou, G., et al. 1984, ApJ, 283, L1

Stanford, S. A., \& Balcells, M. 1990, ApJ, 355, 59

Stanford, S. A., \& Balcells, M. 1991, ApJ, 370, 118

Sturm, E., Lutz, D., Genzel, R., et al. 1996, A\&A, 315, L133

Van der Werf, P. P., Genzel, R., Krabbe, A., et al. 1993, ApJ, 405, 522

Vanzi, L., \& Rieke, G. H. 1997, ApJ, 479, 694

Veron-Cetty, M., \& Veron, P. 1985, A\&A, 145, 425

Vila, M. B., Pedlar, A., Davies, R. D., Hummel, E., \& Axon, D. J. 1990, MNRAS, 242, 379

Yuan, T.-T., Kewley, L. J., \& Sanders, D. B. 2010, ApJ, 709, 884 